Cómo citar / How to cite: López García. J. J. 2020. Fortificaciones, recintos ciclópeos y proceso de romanización en la comarca natural de La Serena (Siglos II a. C. al I d. C.). Mayoral, Victorino. Instituto de Arqueología de Mérida, Mytra 2, Mérida, 2018, 304 pp. ISBN: 978-84-09-03000-2. Antigüedad y Cristianismo 37, 159-163. https:/doi.org/10.6018/ ayc. 458701

\title{
FORTIFICACIONES, RECINTOS CICLÓPEOS Y PROCESO DE ROMANIZACIÓN EN LA COMARCA NATURAL DE LA SERENA (Siglos II A. C. Al I D. C.). MAyoral, Victorino. Instituto de Arqueología de Mérida, MYtra 2, MéridA, 2018, 304 PP. ISBN: 978-84-09-03000-2
}

Recibido: $12-8-2020$

Aceptado: 21-11-2020

El autor de esta monografía desarrolla sus proyectos de investigación dentro del Instituto de Arqueología en Mérida, perteneciente al Consejo Superior de Investigaciones Científicas (CSIC) donde ocupa la plaza de Científico Titular. Victorino Mayoral es uno de los principales abanderados del empleo de métodos no invasivos en el trabajo arqueológico. La continua innovación ha sido una de las principales características de sus trabajos cuyo principal eje vertebrador es el proceso de romanización del sudoeste peninsular. Esta temática ha sido afrontada desde diversas perspectivas, siendo sus trabajos más destacados los abordados desde la Arqueología del Paisaje. Es aquí, donde la aplicación de las Tecnologías de Información Geográfica (TIG) han jugado un papel protagonista, así como el estudio de los paisajes agrarios.

El ámbito geográfico estudiado se corresponde con la comunidad autónoma de Extremadura, destacando los proyectos de Medellín y de la comarca de La Serena. Finalmente, el último de estos proyecto ha sido el de documentación y estudio de las fortificaciones y otras estructuras presentes en la comarca natural de La Serena cuyo resultado final se verá a lo largo de esta monografía.

La obra se compone de 5 capítulos contando cada uno de ellos con varios subcapítulos y epígrafes. Se puede dividir la totalidad de la monografía en tres grandes partes: la primera se corresponde con los antecedentes y los debates historiográficos que giran en torno a los recintos y fortificaciones ciclópeas en 5 zonas geográficas, bien definidas, de la Península Ibérica. Desde esta base se emprende el estudio del caso de la comarca de La Serena sin dejar atrás una extendida explicación de la metodología empleada que, junto a un catálogo de los sitios estudiados, da forma a la segunda parte. La tercera, y última parte, es el resultado del análisis comparativo entre los casos de La Serena y los vistos en la primera parte, junto a un profundo análisis científico donde el empleo de las TIG juega un papel importante. De esta forma, se fijarán dos grandes modelos de ocupación (fortificaciones de altura y recintos de llano) con sus respectivas características que los definen. Dando como resultado la propuesta definitiva de este proyecto, estableciendo la relación de estos modelos con el proceso de romanización del ámbito geográfico estudiado.

En cuanto al análisis estético hay que destacar la rica variedad de fotografías, mapas y otras ilustraciones que dan un gran apoyo gráfico al lector. Si este requiriese de más información puede consultar el DVD que se incluye en la monografía donde se puede ver la totalidad de fotografías, planimetrías y levantamientos topográficos que por razones 
Fortificaciones, recintos ciclópeos y proceso de romanización en la comarca natural de La Serena (Siglos II a. C. al I d. C.). Mayoral, Victorino. Instituto de Arqueología de Mérida, Mytra 2, Mérida, 2018, 304 pp.

editoriales no han podido ser incluidas en la obra impresa.

El tema de la obra, y objetivo principal de este proyecto, es la documentación, estudio e interpretación del gran número de fortificaciones y recintos ciclópeos que se distribuyen por la comarca extremeña de La Serena. No se limita a la mera descripción de estas estructuras, sino que alejándose de un método puramente positivista, se emprende un completo análisis científico que trate de poner fin al debate historiográfico surgido en torno a estas construcciones, polarizado en dos visiones: una poscolonialista que relaciona este fenómeno con la pervivencia de la cultura indígena mediante tratados con Roma, frente a una visión militarista en la que estas construcciones se corresponderían con destacamentos legionarios o auxiliares que asegurarían el control de las vías de comunicación y delos recursos delazona. Como se puede observar de este debate, el fenómeno ligado a la técnica constructiva ciclópea destaca por ser un proceso muy complejo que suscita múltiples interpretaciones.

El capítulo I titulado "Antecedentes sobre el estudio de los recintos y fortificaciones ciclópeas" (pp. 19-40) pretende introducirnos a este modelo de ocupación del territorio. Para ello el autor establece cinco zonas geográficas (Andalucía, el Sureste peninsular, Levante y la Comunidad Valenciana; Cataluña, Aragón y la Meseta; Portugal) sobre las que irá describiendo, de forma sintetizada, los distintos casos de construcciones de aparejo ciclópeo y las teorías que han ido surgiendo en torno a los mismos.

Así, se trata desde el primer capítulo de hacer saber al lector que se encuentra ante un caso de estudio que destaca por su heterogeneidad, un rasgo que provoca la aparición de diversas realidades e interpretaciones en función del área geográfica que se estudie. Andalucía es la primera zona donde el estudio de las fortificaciones cobra una importancia significativa surgiendo las primeras interpretaciones, en los años 70 del pasado siglo, que las relacionan con el control del territorio por parte de la población local. No será hasta décadas más tarde, en 2004, que se señalará la cronología romana de muchos de estos yacimientos, al mismo tiempo, se les atribuirá un marcado carácter multifuncional que va desde ser el centro de explotaciones agrarias hasta el control de la explotación minera. De estos estudios se derivarán diversos intentos de clasificación que serán aprovechados por nuestro autor para sentar las bases de su estudio, estableciendo una primera diferenciación entre hábitats en llano y hábitats en altura. Continuando este recorrido geográfico vemos como el Sureste destaca por la presencia de algunas fortificaciones de carácter puramente militar. Mientras, en la costa alicantina, tras un profundo proceso de revisión, muchos poblados ibéricos han resultado ser fortificaciones vinculadas al control marítimo durante las guerras sertorianas. En el caso de Cataluña no asistimos a unas fortificaciones aisladas, como en los ejemplos anteriores, al mismo tiempo Aragón destaca por sus casas-torre de tradición indígena. Pero sin lugar a duda, el área geográfica que más destaca es el Alentejo portugués que acoge una de las mayores concentraciones de torres y recintos ciclópeos de toda la Península Ibérica siendo uno de los protagonistas del debate historiográfico de sobra conocido.

Una vez conocido el contexto general de este fenómeno ciclópeo el autor nos introduce de lleno en "el caso de la Comarca de La Serena", como indica el título que recibe el capítulo II (pp. 43-72). Para conocer el proceso mental que lleva a la construcción de estos hábitats tan característicos se debe afrontar desde distintas perspectivas, el autor comienza por lo más elemental: el paisaje. Del estudio espacial surgen dos tipos de paisaje enfrentados que condicionan la historia agraria de la comarca: llanura/penillanura vs sierra. La actividad productiva no es el único factor que se ve condicionado, sino que dependiendo del paisaje encontraremos un tipo de construcción 
determinado. Cómo denominar a estas construcciones es un debate que se remonta al propio inicio del estudio de las construcciones ciclópeas, en los años 60 del siglo pasado. El primero en establecer una clasificación es Alonso Rodríguez quien diferencia dos grandes grupos: fortificaciones y recintos de altura (vinculados al control territorial) por un lado, y recintos - torre (cuenta con estructuras turriformes aunque su ubicación no favorezca el control) por el otro. Será Ortiz quien, apoyándose en estudios similares realizados en el Bajo Alentejo por M. Maia, propondrá una nueva clasificación en: torres o recintos torre, recintos en altura, recintos en peñones y las fortificaciones.

Los rasgos de estas construcciones llevarán a hablar sobre una naturaleza militar basándose en el caso de Hijovejo, cuya localización estratégica es una de las claves que sustenta esta hipótesis. Según la cual, este núcleo respondería a las guerras sertorianas y al interés por el control de las minas del sureste extremeño y la transformación de sus minerales por parte del ejército (p. 55). Es en este mismo capítulo donde Mayoral expone su metodología, que se ha ido enriqueciendo científicamente con el paso de los años como es lógico al tratarse de un proyecto de larga duración. Todo comenzó, cuenta el autor, con un intento de contextualizar el territorio de Cancho Roano. Conforme el proyecto avanzaba la primera labor era conocer la localización exacta de estos asentamientos. Una vez encontrado un asentamiento, se procedía a su registro siendo "la principal técnica empleada la realización de levantamientos en modo cinemático en tiempo real" (p. 59). También se apoyan en las fotografías aéreas realizadas desde paramotores hasta drones, con el objetivo de crear modelos fotogramétricos 3D. Pero esto no significa que se omitieran las prospecciones, sino que en los sitios que fue posible se realizaron prospecciones geofísicas, además se emplearon diversas técnicas como el estudio de las cuencas visuales, rutas óptimas, análisis de la topografía local... Atendiendo a este apartado metodológico se puede vislumbrar la calidad científica de este proyecto.

En el inicio del capítulo III "El catálogo de los sitios" (pp. 75-250) se incide, de nuevo, en la vaguedad conceptual que atañe al debate surgido en torno a las fortificaciones y recintos ciclópeos. Por esta razón Mayoral propone una nueva clasificación que, con el apoyo de esta monografía, resulte definitiva y sea tomada por otros investigadores como la base de los nuevos trabajos que surjan en el futuro acerca de esta temática. Alejándose de categorizaciones complejas, que dificulten la adscripción de nuevos sitios arqueológicos a esta clasificación, decide crear dos grupos: fortificaciones en altura y recintos ciclópeos o estructuras de llano, siendo los primeros los ubicados en la sierra y los segundos en penillanura ( $\mathrm{p}$. 75). Este catálogo se compone de 68 casos repartidos por toda la comarca de La Serena, divididos en las siguientes áreas geográficas: el Valle del Guadámez (pp. 78-121), el Valle del Ortigas (pp. 122-183), el Valle del Guadalefra (pp. 184-229), el curso alto del Zújar (pp. 230235) y el curso bajo del Zújar (pp. 236-244). Para facilitar la comparación entre los distintos casos el catálogo cuenta con un elemento descriptivo homogéneo, cuyas partes son las siguientes: descripción de la localización, superficie, estructuras identificadas, valoración cronológica, intervenciones realizadas y referencias bibliográficas.

El capítulo IV “Discusión” (pp. 253-300) cumple con lo que su estoico título promete. Partiendo de la separación entre fortificaciones de altura y recintos de llano, este apartado profundiza en ambos tipos analizando diversas variables que ayuden a plantear una interpretación para explicar la función de estas construcciones. Comenzando por las pautas de localización, se observa que las fortificaciones de altura se encuentran en puntos elevados y de difícil acceso, sin embargo, al contrario de lo que cabría esperar, no son lugares desde los que se domine el paisaje circundante. De igual forma, aprovechan las defensas naturales para la vigilancia de las zonas de paso entre las 
Fortificaciones, recintos ciclópeos y proceso de romanización en la comarca natural de La Serena (Siglos II a. C. al I d. C.). Mayoral, Victorino. Instituto de Arqueología de Mérida, Mytra 2, Mérida, 2018, 304 pp.

sierras que copan su orografía. Finalmente, en cuanto a su organización interna, presentan "una fuerte compartimentación del espacio" (p. 256). A diferencia de lo anterior, los recintos de llano destacan por su disposición homogénea y por ubicarse sobre pequeñas elevaciones desde las que se camuflan a larga distancia, por lo que se presume que no tienen la misma función de control que las fortificaciones.

El segundo epígrafe se corresponde con las técnicas constructivas, la cuarcita es la principal materia prima pues es el recurso con mayor disponibilidad. En el caso de las fortificaciones el material se extrae del propio lugar donde se va a construir, pero esto no omite el hecho de que se emplean técnicas constructivas muy sofisticadas. En palabras del autor: "Se emplea una mampostería de grandes bloques poligonales, encajados a seco con la ayuda de cuñas de menor tamaño" (p. 259). Se trata de muros de doble hilada que se apoyan sobre la propia base geológica, sus alzados son paredes de adobe o tapial, cuentan con pavimentos de tierra batida y las techumbres son de madera o cubiertas vegetales. Lo más característico de los recintos de llano es el "ciclopeísmo de los paramentos" (p. 269). Otros elementos por señalar son sus torres de mampostería en seco, en ocasiones sillarejo, que no se documentan zanjas de cimentación, sus alzados de tierra y las cubiertas de tegulae.

En cuanto a sus dimensiones y organización del espacio Mayoral nos dice lo siguiente de las fortificaciones de altura: "Un perímetro cerrado con una o varias líneas de murallas, complementadas por la defensa natural de la roca, con uno o dos puntos de acceso y en su parte más alta una o varias construcciones de gran aparejo y más de una planta, que actuarían como torres o atalayas" (p. 261). Al mismo tiempo, clasifica este tipo de construcciones atendiendo a sus dimensiones en tres grupos: las fortificaciones de $300 \mathrm{~m} 2-700 \mathrm{~m} 2,700 \mathrm{~m} 2$ $-1000 \mathrm{~m} 2$ y en torno a $1500 \mathrm{~m} 2$. El análisis, en este sentido, de los recintos de llano es más simple pues responden a un mismo esquema equitativo siendo lo más característico la presencia de una estructura presumiblemente turriforme.

La cronología de ambos modelos es muy similar abarcando del s. II a.C al I d.C, de aquí que el autor afirme que existe una vinculación entre la arquitectura ciclópea y una ocupación plenamente romana (p. 279). Del último punto de análisis se desprende que mientras las fortificaciones presentan una baja explotación del entorno, aunque muchas de ellas serían autosuficientes, los recintos de llano serían centros de almacenaje, transformación y consumo de alimentos, de lo que se interpreta la existencia de una producción de ámbito regional.

El capítulo IV cierra con los resultados del análisis espacial de las localizaciones (pp. 288-300) en virtud de la altitud relativa y otros indicadores de la topografía local. También de los cálculos de visibilidad, según los cuales las fortificaciones ofrecen una excelente cobertura visual sobre los corredores naturales entre sierras, y del análisis del movimiento, cuyo resultado resume Mayoral en la siguiente afirmación: "Hay una serie de fortificaciones de altura cuya localización parece explicarse en función de los ejes preferentes de movimiento en sentido longitudinal a lo largo de los corredores entre las sierras, mientras que otros aparecen claramente distribuidos en función de las rutas transversales que conectan estos pasillos entre sí" (p. 299).

Finalmente, el capítulo V titulado "Conclusiones. Los recintos en su contexto histórico: el SE extremeño entre el s. II a. C. y el I d. C." (pp. 303-322) trata de establecer una relación definitiva entre estos modelos constructivos y el devenir del proceso histórico del contexto geográfico que ocupan. De esta manera, vemos como el oppidum de Magacela actúa como eje vertebrador de gran parte de estos recintos ciclópeos. Estos serían el resultado del colapso del modelo de ocupación anterior basado en edificios señoriales, de influencia orientalizante, por lo que no contarían con antecedentes indígenas. La importancia de Magacela explicaría las 
escasas evidencias de ocupación republicana en Metellinum (Medellín), a pesar de la teórica importancia de este núcleo que no alcanzará un estatus privilegiado hasta época cesariana o augustea. La Comarca de La Serena será una zona muy inestable hasta mediados del s. I a. C. (p. 307) pues supone una encrucijada de las principales vías de comunicación que se dirigían hasta Corduba.

Del análisis de las fortificaciones de altura se traduce que su construcción parece responder a la labor continua de un mismo equipo de constructores de ahí que la técnica constructiva refleje una mentalidad netamente romana, aunque hay "un papel destacado del elemento indígena en el surgimiento del sistema de control" (p. 313). Por lo tanto, el autor concluye que: "La vigilancia y control de paso, unido a la potencial disuasión del uso de la fuerza, parecen estar pues detrás de la red de fortificaciones de altura" (p.313).

En relación con los recintos de llano se vislumbran dos escenarios: enclaves relacionados con actividades agrícolas y recintos ocupados por grupos de elevado poder adquisitivo, como indica la presencia de vajilla itálica de gran calidad, si bien podrían mantener su función como atalayas de vigilancia. Aunque, como asegura Mayoral unas páginas después: "los recintos ciclópeos como asentamientos de carácter rural, más ligados a la explotación del entorno que a la vigilancia y el control de recursos o caminos" (p. 315). Por esta razón, se puede concluir que los recintos ciclópeos son el primer paso hacia una ocupación generalizada de las tierras. Una vez concluida la lectura de esta obra puede dar la impresión de que se trata de un proyecto finalizado, pero nada más lejos de la realidad. Todavía son necesarios multitud de análisis desde la perspectiva de la Arqueología del Paisaje para buscar respuesta a los interrogantes que han quedado abiertos. Aun así, no quita que nos encontremos ante un trabajo de excelente calidad científica e innovador gracias al uso de las TIG, factores que lo convierten en una obra de referencia para el estudio de las fortificaciones y los recintos ciclópeos en el proceso de romanización de la Península Ibérica.

\author{
Juan José López García \\ Universidad de Murcia, \\ Murcia, España \\ juanjose.lopezgarcia4@gmail.com \\ orcid.org/0000-0001-8804-6158
}


